\title{
RECEDING HORIZON OPTIMAL CONTROL FOR INFINITE DIMENSIONAL SYSTEMS
}

\author{
KaZufumi Ito $^{1}$ And KarL KunisCH ${ }^{2}$
}

\begin{abstract}
The receding horizon control strategy for dynamical systems posed in infinite dimensional spaces is analysed. Its stabilising property is verified provided control Lyapunov functionals are used as terminal penalty functions. For closed loop dissipative systems the terminal penalty can be chosen as quadratic functional. Applications to the Navier-Stokes equations, semilinear wave equations and reaction diffusion systems are given.
\end{abstract}

Mathematics Subject Classification. 49L15, 49N35, 93C20, 93Dx.

Received January 14, 2002.

\section{INTRODUCTION}

We consider the following optimal control problem in Hilbert spaces $X$ and $W$ : minimize the performance index

$$
\int_{0}^{T_{\infty}} f^{0}(x(t), u(t)) \mathrm{d} t
$$

subject to

$$
\frac{\mathrm{d}}{\mathrm{d} t} x(t)=f(x(t), u(t)), \text { for } t>0, x(0)=x_{0}, \quad \text { and } u(t) \in U
$$

Here $U$ is a closed convex subset of $W$. We refer to $x(\cdot)$ and $u(\cdot)$ as state and control functions with $x(t) \in X$ and $u(t) \in U$.

For the purpose of this introductory discussion we assume that for every $x_{0} \in X$ and $u \in \mathcal{U}_{\mathrm{ad}}=\{u \in$ $L_{\text {loc }}^{2}(0, \infty ; W): u(t) \in U$ a.e. $\}$ there exists an $X$-valued continuous semi-flow $x(t)=x\left(t ; x_{0}, u\right)$ which is a weak solution to $(1.2)$. Under appropriate conditions $(1.1,1.2)$ admits a solution which satisfies the minimum

Keywords and phrases: Receding horizon control, control Lyapunov function, Lyapunov equations, closed loop dissipative, minimum value function, Navier-Stokes equations.

1 Department of Mathematics, North Carolina State University, Raleigh, North Carolina, USA.

Research partially supported by National Science Foundation under grant UINT-8521208.

2 Institut für Mathematik, Karl-Franzens-Universität Graz, 8010 Graz, Austria; e-mail: karl.kunisch@uni-graz.at Research partially supported by the Fonds zur Förderung der wissenschaftlichen Forschung under SFB 03 "Optimierung und Kontrolle". 
principle

$$
\left\{\begin{array}{l}
\frac{\mathrm{d}}{\mathrm{d} t} x(t)=H_{p}(x(t), u(t), p(t)), x(0)=x_{0}, \\
\frac{\mathrm{d}}{\mathrm{d} t} p(t)=-H_{x}(x(t), u(t), p(t)), p\left(T_{\infty}\right)=0, \\
u(t)=\arg \min _{u \in U} H(x(t), u, p(t),
\end{array}\right.
$$

where $H$ is the Hamiltonian defined by $H(x, u, p)=f^{0}(x, u)+(p, f(x, u))_{X}$. The coupled system of two-point boundary value problems with initial condition for the primal equation and terminal condition for the adjoint equation represents a significant challenge for numerical computations in case $T_{\infty}$ is large and it has therefore been the focus of many research efforts. An alternative is to construct the feedback solution based on Bellman's dynamic programming principle but again, due to computational costs, this is not tractable except for very limited examples.

In view of the difficulties explained above the question of obtaining suboptimal controls arises. One of the possibilities is the time-domain decomposition by receding horizon formulations [1]. Receding horizon techniques have proved to be effective numerically both for optimal control problems governed by ordinary (e.g. $[3,11-13,15,16])$ and for partial differential equations, e.g. in the form of the instantaneous control technique for problems in fluid mechanics $[2,4,5,9]$.

To briefly explain the strategy let $0=T_{0}<T_{1} \ldots<T_{n}=T_{\infty}$ describe a grid on $\left[0, T_{\infty}\right]$ and let $T \geq$ $\max \left\{T_{i+1}-T_{i}: i=0, \ldots, n-1\right\}$. The receding horizon optimal control problem involves the successive finite horizon optimal control on $\left[T_{i}, T_{i}+T\right]$ :

$$
\min \int_{T_{i}}^{T_{i}+T} f^{0}(x(t), u(t)) \mathrm{d} t+G\left(x\left(T_{i}+T\right)\right),
$$

subject to

$$
\frac{\mathrm{d}}{\mathrm{d} t} x(t)=f(x(t), u(t)), t \geq T_{i}, \quad x\left(T_{i}\right)=\bar{x}\left(T_{i}\right),
$$

where $\bar{x}$ is the solution to the auxiliary problem on $\left[T_{i-1}, T_{i-1}+T\right]$. If $T>T_{i+1}-T_{i}$ we have overlapping domains. The solution on $\left[0, T_{\infty}\right]$ is obtained by concatenation of the solutions on $\left[T_{i}, T_{i+1}\right]$ for $i=0, \ldots, n-1$. If $x\left(T_{i}\right)$ is observed, then the receding horizon control is a state feedback since the control on $\left[T_{i}, T_{i+1}\right]$ is determined as a function of the state $\bar{x}\left(T_{i}\right)$. The optimal pair $\left(x\left(t-T_{i}\right), u\left(t-T_{i}\right)\right), t \in\left[T_{i}, T_{i}+T\right]$ satisfies the two point boundary value problem (1.3) on the interval $[0, T]$ with the terminal condition $p(T)=G_{x}(x(T))$ and the initial condition $x(0)=\bar{x}$. System (1.3) with $T_{\infty}$ replaced by $T$, with $T$ sufficiently smaller than $T_{\infty}$, is better conditioned and much easier to solve numerically than (1.3) itself.

The theoretical justification of receding horizon control techniques can be addressed by means of the stabilization problem. Assuming that $x=0$ is a steady state for (1.2) with $u=0$ which can be stabilized by means of an optimal control formulation (1.1) with $T_{\infty}=\infty$. The question is addressed whether stabilization can also be achieved by means of a receding horizon synthesis. In order to establish asymptotic stability of the receding horizon control we utilize a terminal penalty term $G\left(x\left(T_{i}+T\right)\right)$ rather than terminal constraints requiring that $x\left(t_{i}+T\right)$ is contained in an appropriate neighborhood of the origin which is frequently used for receding horizon control in connection with ordinary differential equations, see $e . g$. [14]. The functional $G: X \rightarrow R$ will be chosen as an appropriately defined control Lyapunov function, see Definition 2.1 below. It will be shown that the incorporation of the terminal cost $G$ to the cost functional provides asymptotic stability and that a suboptimal synthesis for minimizing (1.1) by receding horizon control.

Control Lyapunov functions received a considerable amount of attention as a means of analyzing the stability of the control system $(1.1,1.2)$, regardless of issues related to optimal control. We refer to the monograph [6] 
and the references given there. The use of control Lyapunov functions within the context of receding horizon control is a recent one. In [15] control Lyapunov functions were utilized as explicit constraints in the auxiliary problems to guarantee that the final state $x\left(T_{i}+T\right)$ lies within the level curve of the control Lyapunov function that is determined by the trajectory controlled by a minimum norm control. The analysis in [11] utilizes control Lyapunov functions as a terminal penalty as in (1.4). The stabilizing properties of the resulting receding horizon optimal control strategy are analyzed under the assumption that $f$ possesses an exponentially stabilizable critical point.

Let us now outline the contributions of this paper. In Section 2 we introduce and discuss a control Lyapunov function $G$ (see Def. 2.1 and Th. 2.1), and then we establish monotonicity of the value function $V_{T}\left(x_{0}\right)$ :

$$
V_{T}\left(x_{0}\right)=\inf \left\{\int_{0}^{T} f^{0}(x(t), u(t)) \mathrm{d} t+G(x(T)) \text { subject to }(1.2)\right\}
$$

with respect to $T$, i.e., $V_{T}\left(x_{0}\right) \leq V_{\hat{T}}\left(x_{0}\right) \leq G\left(x_{0}\right)$ for $0 \leq \hat{T} \leq T$ and $x_{0} \in X$, provided that $G$ is a control Lyapunov function. This will imply (see, Ths. 2.2-2.4) that

$$
\left.G\left(x_{i+1}\right)\right)+\int_{T_{i}}^{T_{i+1}} f^{0}(\bar{x}(t), \bar{u}(t)) \mathrm{d} t \leq G\left(x_{i}\right)
$$

where $x_{i}=\bar{x}\left(T_{i}\right)$. This implies that the states $x_{i}$ are confined to the level set $S_{\alpha}=\left\{x \in X: G(x) \leq G\left(x_{0}\right)=\alpha\right\}$. Assume that $f(0,0)=0$ and $G(0)=0$ and that $f^{0}(x, u)>0$ and $G(x)>0$ except at $(0,0)$. Then, we have $G\left(x_{i+1}\right)<V_{T}\left(x_{i}\right) \leq G\left(x_{i}\right)$. If we assume orbit compactness then for $0 \neq x_{0} \in S_{\alpha}$ we have $G\left(x_{i+1}\right) \leq \rho G\left(x_{i}\right)$ for some $\rho<1$. Hence $G\left(x_{k}\right) \leq \rho^{k} G\left(x_{0}\right) \rightarrow 0$ as $k \rightarrow \infty$, which implies asymptotic stability. Moreover, if $f^{0}(x, u) \geq \omega G(x)$ for some $\omega>0$, then $G\left(x_{i+1}\right) \leq \mathrm{e}^{-\omega T} G\left(x_{i}\right)$ (see, Th. 2.4). In Sections 3 and 4 we formulate the control problem $(1.1,1.2)$ in a Gelfand triple formulation and as semi-linear control systems respectively. We apply our formulations to concrete examples including the incompressible Navier-Stokes equations and a semilinear damped wave equation. We also investigate the important special situation when the quadratic functional $G(x)=\frac{\alpha}{2}|x|^{2}, \alpha>0$, can be chosen as a control Lyapunov function. This is the case if the control system (1.2) is closed loop dissipative, see Definition 3.1, which is useful for certain classes of dissipative equations.

In general this standard quadratic form is not a control Lyapunov function. In Section 4 we analyze a quadratic form motivated from energy multipliers for the semi-linear wave equations and show that it defines a control Lyapunov function. Under the assumption that the linear part of (1.2) is stabilizable by linear feedback, we give in Section 5 the construction of a quadratic form based on the Lyapunov equation and show that it defines a local control-Lyapunov function. We also provide an analysis for the choice of the terminal cost based on finite dimensional approximations to the infinite dimensional control Lyapunov function.

In our discussion above it is assumed that the infinite time horizon optimal control problem admits a solution, which holds true, for example, in the case of stabilizable steady states. In general this assumption may not satisfied. Consider, for instance disturbance attenuation problems and problems with cost functionals of tracking type. As in the case of finite dimensional control problems [10] the results in this paper can be extended to such cases by introducing a control $\lambda$-Lyapunov function, where the positive constant $\lambda$ represents the attenuation or tracking rate.

The receding horizon formulation requires knowledge of the state $x\left(T_{i}\right)$ to employ it in feedback form. In the case of partial observations one can construct a state observer system to estimate $x\left(T_{i}\right)$ based on the linearization of the state dynamics about the optimal pair. This will be discussed in forthcoming work. Finally we mainly treated bounded distributed affine controls. We aim for extending our analysis to boundary control and bilinear control problems. 


\section{LOCAL CONTROL LyAPUNOV FUNCTIONS}

Let $X$ and $W$ be Hilbert spaces representing the state and the control space for the autonomous control system

$$
\left\{\begin{array}{l}
\frac{\mathrm{d}}{\mathrm{d} t} x(t)=f(x(t), u(t)), t>0 \\
x(0)=x_{0}
\end{array}\right.
$$

with $f(0,0)=0$. Further let $U$ be a closed convex subset of $W$ and, for $T>0$, set

$$
\mathcal{U}_{\mathrm{ad}}=\left\{u \in L_{\mathrm{loc}}^{2}(0, \infty ; W): u(t) \in U \text { for a.e. } t \in(0, \infty)\right\}
$$

For $T \in(0, \infty]$ and $u \in \mathcal{U}_{\text {ad }}$ we refer to $x=x\left(\cdot ; x_{0}, u\right)$ as solution to $(2.1)$ on $[0, T)$ if

$$
\left\{\begin{array}{l}
x\left(0 ; x_{0}, u\right)=x_{0}, \\
x\left(t+s ; x_{0}, u\right)=x\left(t ; x\left(s ; x_{0}, u\right), u(\cdot+s)\right), \\
\text { for all } 0 \leq s, 0 \leq t, 0 \leq t+s \leq T .
\end{array}\right.
$$

Contents permitting the dependence of $x$ on $x_{0}$ and $u$ will be suppressed. Throughout we assume that for every $x_{0} \in X$ and $T>0$ there exists a control $u \in L^{2}(0, T ; U)$ such that $(2.1)$ admits a solution $x=x\left(\cdot, x_{0}, 0\right)$ on $[0, T]$. When referring to the receding horizon strategy we shall for the sake of simplicity assume that the grid is uniform and that $t_{k}=k T$ for $k=0, \ldots$

The optimal control problems are defined next. Let

$$
f^{0}: X \times U \rightarrow R^{+}
$$

$R^{+}=\{r \geq 0\}$, denote a continuous function and consider the infinite horizon problem

$$
\inf _{u \in \mathcal{U}_{\mathrm{ad}}} \int_{0}^{\infty} f^{0}(x(t), u(t)) \mathrm{d} t \quad \text { subject to }(2.1)
$$

If $f^{0}$ is quadratic and positive definite in $x$ and $u$ then (2.3) represents a stabilization problem for (2.1). As described in the introduction the receding horizon strategy consists of a sequence of subproblems with control horizon of length $T$. The building blocks of the strategy are given by the problems

$$
\left\{\begin{array}{l}
\inf _{u \in \mathcal{U}_{\text {ad }}} \int_{0}^{T} f^{0}(x(t), u(t)) \mathrm{d} t+G(x(T)) \\
\text { subject to }(2.1) .
\end{array}\right.
$$

Here $G: X \rightarrow R^{+}$is chosen as local control Lyapunov function which is defined next.

Definition 2.1. A nonnegative continuous functional $G: X \rightarrow R$ with $G(0)=0$ is called local control Lyapunov functional (CLF) for (2.3) if there exists $\alpha>0$ such that for all $x_{0} \in S_{\alpha}=\{x \in X: G(x) \leq \alpha\}$ and $T>0$ there exists a control $u=u\left(\cdot ; x_{0}, T\right) \in \mathcal{U}_{\text {ad }}$ such that

$$
\int_{s}^{T} f^{0}(x(t), u(t)) \mathrm{d} t+G(x(T)) \leq G(x(s)), \text { for } 0 \leq s \leq T,
$$

where $x$ is a solution to (2.1). $G$ is called global CLF if (2.5) holds for all $x_{0} \in X$. 
To investigate the receding horizon strategy we introduce the value functionals for the infinite and the finite horizon problems (2.3) and (2.4):

$$
V_{T}\left(x_{0}\right)=\inf _{u \in \mathcal{U}} \int_{0}^{T} f^{0}(x(t), u(t)) \mathrm{d} t+G(x(T)),
$$

subject to (2.1) and

$$
V\left(x_{0}\right)=\inf _{u \in \mathcal{U}_{\mathrm{ad}}} \int_{0}^{\infty} f^{0}(x(t), u(t) \mathrm{d} t
$$

subject to $(2.1)$

Theorem 2.1. Suppose that $G$ is a local CLF for (2.3), and $T>0, x_{0} \in S_{\alpha}$. Then we have $V_{T}\left(x_{0}\right) \leq G\left(x_{0}\right)$ and $V\left(x_{0}\right) \leq G\left(x_{0}\right)$. If in addition $G$ is a global $C L F$ then $V\left(x_{0}\right) \leq V_{T}\left(x_{0}\right)$ for all $x_{0} \in X$.

Proof. The first inequality is a consequence of the definition of local CLF. We also have $G(x(T)) \leq G\left(x_{0}\right)$ and hence $x(T) \in S_{\alpha}$. Therefore concatenation of the solutions arising from repeated applications of (2.6) on the intervals $[(k-1) T, k T]$ allows to construct a control $u \in \mathcal{U}_{\text {ad }}$ with associated solution $x=x(\cdot ; u)$ such that for each $k \geq 1$

$$
G(x(k T))+\int_{(k-1) T}^{k T} f^{0}(x(t), u(t)) \mathrm{d} t \leq G(x((k-1) T)) .
$$

Summation over $k$ implies that

$$
G(x(k T))+\int_{0}^{k T} f^{0}(x(t), u(t)) \mathrm{d} t \leq G\left(x_{0}\right) .
$$

By the Lebesgue-Fatou lemma and non-negativity of $f^{0}$ we have

$$
\int_{0}^{\infty} f^{0}(x(t), u(t)) \mathrm{d} t \leq G\left(x_{0}\right)
$$

and hence $V\left(x_{0}\right) \leq G\left(x_{0}\right)$. Utilizing the properties for $x$ to be a solution of (2.1) as specified in (2.2) allows to employ the optimality principle

$$
V\left(x_{0}\right)=\inf _{u \in \mathcal{U}_{\mathrm{ad}}}\left\{\int_{0}^{T} f^{0}(x(t), u(t)) \mathrm{d} t+V(x(T))\right\},
$$

where $x=x\left(\cdot ; x_{0}, u\right), e . g$. , see [7]. If $G$ is a global CLF then by the argument above $V(x(T)) \leq G(x(T))$. Combined with (2.6) this implies $V\left(x_{0}\right) \leq V_{T}\left(x_{0}\right)$.

Theorem 2.2 (Monotonicity). Let $G$ be a local $C L F$ and $0 \leq \hat{T} \leq T$. Then we have

$$
V_{T}\left(x_{0}\right) \leq V_{\hat{T}}\left(x_{0}\right) \text { for all } x_{0} \in S_{\alpha}
$$

Proof. Let $\delta>0$ be arbitrary. Then there exists $(\bar{x}, \bar{u})$ with $\bar{u} \in L^{2}(0, \hat{T} ; U)$ and $\bar{x}$ a solution to $(2.1)$ on $[0, \hat{T}]$ such that

$$
\int_{0}^{\hat{T}} f^{0}(\bar{x}(t), \bar{u}(t)) \mathrm{d} t+G(\bar{x}(\hat{T})) \leq V_{\hat{T}}\left(x_{0}\right)+\delta .
$$


We need to argue that $\bar{u}$ can be chosen such that $G(\bar{x}(\hat{T})) \leq \alpha$. For this purpose note that $V_{\hat{T}}\left(x_{0}\right) \leq G\left(x_{0}\right)$ by Theorem 2.1. If $V_{\hat{T}}\left(x_{0}\right)=G\left(x_{0}\right)$ then by the definition of CLF $\bar{u}$ can be chosen such that $G(\bar{x}(\hat{T})) \leq G\left(x_{0}\right)$ $=V_{\hat{T}}\left(x_{0}\right) \leq \alpha$. Otherwise $G\left(x_{0}\right)>V_{\hat{T}}\left(x_{0}\right)$ and choosing $\delta \leq G\left(x_{0}\right)-V_{\hat{T}}\left(x_{0}\right)$, there exists again $\bar{u}$ such that (2.7) holds and $G(\bar{x}(\hat{T})) \leq G\left(x_{0}\right) \leq \alpha$. By $(2.5)$ there is a control $\tilde{u}=u(\cdot ; \bar{x}(\hat{T}), T-\hat{T})$ such that

$$
\int_{0}^{T-\hat{T}} f^{0}(\tilde{x}(t), \tilde{u}(t)) \mathrm{d} t+G(\tilde{x}(T-\hat{T})) \leq G(\tilde{x}(0)),
$$

where $\tilde{x}=\tilde{x}(\cdot ; \bar{x}(\hat{T}), \tilde{u})$. Concatenation of the solution and control pairs $(\bar{x}, \bar{u})$ and $(\tilde{x}, \tilde{u})$ defines a control $u$ with associated solution $x$ on $[0, T]$ satisfying

$$
V_{T}\left(x_{0}\right) \leq \int_{0}^{T} f^{0}(x(t), u(t)) \mathrm{d} t+G(x(T)) \leq \int_{0}^{\hat{T}} f^{0}(x(t), u(t)) \mathrm{d} t+G(x(\hat{T})) .
$$

Combined with (2.7) this implies that $V_{T}\left(x_{0}\right) \leq V_{\hat{T}}\left(x_{0}\right)+\delta$. Since $\delta>0$ was chosen arbitrarily we have $V_{T}\left(x_{0}\right) \leq V_{\hat{T}}\left(x_{0}\right)$ for $0 \leq \hat{T} \leq T$.

Theorem 2.3. Assume that $G$ is a local $C L F$ and that $(\bar{x}, \bar{u})$ is a solution to the receding horizon problems $(1.4,1.5)$ on $[0, \infty)$. Then we have for every $x_{0} \in S_{\alpha}$ and $k=1,2, \cdots$

$$
G\left(x_{k}\right)+\int_{0}^{k T} f^{0}(\bar{x}(t), \bar{u}(t)) \mathrm{d} t \leq G\left(x_{0}\right) .
$$

If $V_{T}(x) \leq \rho_{T} G(x)$ for some $\rho_{T} \leq 1$ and $T>0$, independently of $x \in S_{\alpha}$, then $G\left(x_{k}\right) \leq \rho_{T}^{k} G\left(x_{0}\right)$ for all $k=1,2, \cdots$ If $G$ is a global CLF then $V_{T}(\bar{x}(t)) \leq V_{T}\left(x_{k-1}\right)$ for all $t \in\left[T_{k-1}, T_{k}\right]$.

Proof. Inequality (2.7) follows from repeated application of (2.5). Note that by construction $x_{k} \in S_{\alpha}$ for all $k$. If $V_{T}(x) \leq \rho_{T} G(x)$ for some $\rho_{T} \leq 1, T>0$, independently of $x \in S_{\alpha}$ then $G\left(x_{k}\right) \leq V_{T}\left(x_{k}\right) \leq \rho_{T} G\left(x_{k-1}\right)$ since $f^{0} \geq 0$ and by iteration $G\left(x_{k}\right) \leq \rho_{T}^{k} G\left(x_{0}\right)$ for each $k=1,2, \cdots$ Utilizing the properties in (2.2) satisfied by a solution to (2.1) allows to apply the optimality principle which implies

$$
V_{T}\left(x_{k-1}\right)=\int_{(k-1) T}^{t} f^{0}(\bar{x}(t), \bar{u}(t)) \mathrm{d} t+V_{k T-t}(\bar{x}(t)), \text { for } t \in[(k-1) T, k T] .
$$

If $G$ is a global CLF then $T \rightarrow V_{T}(\bar{x}(t))$ decays monotonically by Theorem 2.2 and consequently $V_{T}(\bar{x}(t))$ $\leq V_{T}\left(x_{k-1}\right) \leq G\left(x_{k-1}\right)$ for $t \in[(k-1) T, k T]$.

In Section 4 we shall consider a class of problems where $\rho_{T}$ of Theorem 2.3 can be taken strictly small than 1 . Next we give a condition for $\rho<1$ which is applicable in case that the controlled orbits are compact.

Let us define for $\alpha>0$

$$
S=\left\{(x, u) \in X \times L^{2}(0, T ; U): x=x\left(T ; x_{0}, u\right), x_{0} \in S_{\alpha}, G\left(x\left(T ; x_{0}, u\right)\right)+\int_{0}^{T} f^{0}(x, u) \mathrm{d} t \leq G\left(x_{0}\right)\right\},
$$

and set $B_{\delta}=\{x:|x| \leq \delta\}$, for $\delta>0$.

Proposition 2.1. Let $G$ be a local $C L F$ and assume that $S$ is compact in $X \times L^{2}(0, T ; U)_{\text {weak }}, f^{0}(x, u)>0$ for $x \neq 0, G(x)>0$ for $x \neq 0$, and that $\left(x_{0}, u\right) \rightarrow x\left(T ; x_{0}, u\right)$ is continuous from $S \subset X \times L^{2}(0, T ; U)_{\text {weak }}$ to $X$. Then for every $\delta>0$ there exists $\rho=\rho(T, \delta)<1$ such that

$$
G(x(T ; \bar{x}, u)) \leq \rho G(\bar{x}),
$$

for all $(\bar{x}, u) \in S$ with $\bar{x} \notin B_{\delta}$. 
Proof. Let $\bar{x} \in S, \bar{x} \neq 0$, and let $\bar{u}$ denote a control such that (2.5) is satisfied for the associated trajectory. There exists a nontrivial time interval on which the trajectory $x(\cdot ; \bar{x}, u)$ does not vanish and consequently $G(x(T ; \bar{x}, u))<G(\bar{x})$. If the assertion of the theorem is false then there exists a sequence $\left\{\left(\bar{x}_{n}, u_{n}\right)\right\} \in S$ with $\bar{x}_{n} \notin B_{\delta}$ such that

$$
G\left(x\left(T ; \bar{x}_{n}, u_{n}\right)\right) \geq\left(1-\frac{1}{n}\right) G\left(\bar{x}_{n}\right) .
$$

By compactness there exist subsequences of $\left\{\bar{x}_{n}\right\}$ and $\left\{\bar{u}_{n}\right\}$, denoted by the same symbol, and $(\hat{x}, \hat{u}) \in S$ with $\hat{x} \notin B_{\delta}$, such that $\lim \bar{x}_{n}=\hat{x}$ in $X$ and $\lim \bar{u}_{n}=\hat{u}$ weakly in $L^{2}(0, T ; U)$. As a consequence of the continuity assumption $\lim _{n \rightarrow \infty} x\left(T ; \bar{x}_{n}, \bar{u}_{n}\right)=\hat{x}$ and hence $G(x(T ; \hat{x}, \hat{u})) \geq G(\hat{x})$, which is impossible.

Proposition 2.1 asserts that orbits originating in $S_{\alpha}$ and controlled by the receding horizon strategy decay into arbitrary small neighborhoods of the origin with a uniform decay rate.

Theorem 2.4 (Stability). Assume that $G$ is a global $C L F$ and that $f^{0}(x, u) \geq \omega G(x)$ for some $\omega>0$ and all $x \in X$ and $u \in U$, and that $(u(t), x(t))$ minimizes $\int_{0}^{T} f^{0}(x(t), u(t)) \mathrm{d} t+G(x(T))$ over $u \in U_{\text {ad }}$ subject to (2.1). Then we have

$$
G(x(T)) \leq \mathrm{e}^{-\omega T} G\left(x_{0}\right)
$$

Proof. By the optimality principle

$$
\int_{t}^{\tau} f^{0}(x(s), u(s)) \mathrm{d} s+V_{T-\tau}(x(\tau))=V_{T-t}(x(t))
$$

for every $0 \leq t \leq \tau \leq T$. From (2.10) it follows that $t \rightarrow g(t)=V_{T-t}(x(t))$ is a $W^{1,1}$-function. By Theorem 2.1 and the lower bound on $f^{0}$ it follows that

$$
\omega \int_{t}^{\tau} V_{T-s}(x(s)) \mathrm{d} s+V_{T-\tau}(x(\tau)) \leq V_{T-t}(x(t))
$$

and consequently

$$
\omega g(t)+\frac{\mathrm{d}}{\mathrm{d} t} g(t) \leq 0 \text { for a.e. } t \in[0, T]
$$

Multiplying by $\mathrm{e}^{\omega t}$ and integrating on $[0, T]$ implies that

$$
\mathrm{e}^{\omega T} g(T)-g(0) \leq 0
$$

\section{Gelfand triple Formulation}

Let $V \subset X=X^{*} \subset V^{*}$ be a Gelfand triple, $W=U$, and let $f: V \times U \rightarrow V^{*}$ be a continuous mapping. We assume that for every $x_{0} \in X$ and $u \in L_{\text {loc }}^{2}(0, \infty ; U)$ there exists $\tau>0$ (depending on $\left|x_{0}\right|_{X}$ and $\int_{0}^{\tau}|u|^{2} \mathrm{~d} s$ ) such that there exists a unique solution $x=x\left(\cdot ; x_{0}, u\right) \in W(0, \tau)=L^{2}(0, \tau ; V) \cap H^{1}\left(0, \tau ; V^{*}\right)$ satisfying

$$
x(t)-x_{0}=\int_{0}^{t} f(x(s), u(s)) \mathrm{d} s \quad \text { in } V^{*}
$$

and $\left|x(t)-x_{0}\right|_{X} \rightarrow 0$ as $t \rightarrow 0^{+}$. Moreover we assume that $\left|x(t)-x_{0}\right|_{V} \rightarrow 0$ as $t \rightarrow 0^{+}$if $x_{0} \in V$. Note that $W(0, T)$ is continuously embedded into $C(0, T ; X)\left(\right.$ i.e. $\left.|x(t)|_{H}^{2} \leq \int_{0}^{t}\left(|x(s)|_{V}^{2}+\left|\frac{\mathrm{d}}{\mathrm{d} t} x(s)\right|_{V^{*}}^{2}\right) \mathrm{d} t\right)$. 
We shall say that the solutions to (3.1) depend continuously on $x_{0} \in X$ and $u \in L^{2}(0, \tau ; U)$ if for every $C>0$ there exists $\tau>0$ and a continuous, nondecreasing function $M_{C}(t)$ with $M_{C}(0)=1$ such that

$$
\left|x\left(\cdot, x_{0}, u\right)-x\left(\cdot ; y_{0}, v\right)\right|_{W(0, t)}^{2} \leq M_{C}(t)\left(\left|x_{0}-y_{0}\right|_{X}^{2}+\int_{0}^{t}|u(s)-v(s)|^{2}\right)
$$

for all $t \leq \tau, x_{0}, y_{0} \in X$ and $u, v \in L^{2}(0, \tau ; U)$ satisfying

$$
\left|x_{0}\right|,\left|y_{0}\right| \leq C \text { and } \quad \int_{0}^{\tau}|u|^{2} \mathrm{~d} s, \int_{0}^{\tau}|v|^{2} \mathrm{~d} s \leq C^{2}
$$

We have the following relationship between control Lyapunov functions and the Hamilton-Jacobi inequality (3.2) given below.

Theorem 3.1. Assume that $G$ is a convex $C^{1}$-functional on $X$, with $G(0)=0$, and that $x \in V \rightarrow G^{\prime}(x) \in V$ is continuous.

(a) (Necessity) Assume that for every $x \in V$

(i) $u \rightarrow f(x, u)$ is continuous from $U$ endowed with the weak topology to $V^{*}$ and $u \rightarrow f^{0}(x, u)$ is weakly lower semi-continuous,

(ii) $U$ is weakly compact or $u \rightarrow f^{0}(x, u)$ is coercive.

Then, if $G$ is a global control Lyapunov function, there exists for all $x_{0} \in V$ an element $u \in U$ such that

$$
\left\langle G^{\prime}\left(x_{0}\right), f\left(x_{0}, u\right)\right\rangle_{V, V^{*}}+f^{0}\left(x_{0}, u\right) \leq 0
$$

(b) (Sufficiency) Assume that the solutions to (3.1) depend continuously on $x_{0} \in X$ and $u \in L^{2}(0, \tau ; U)$ and that $f$ with $f(0,0)=0$ is continuous in the sense that

$$
f\left(x_{n}, u_{n}\right) \rightarrow f(x, u) \text { weakly in } L^{2}\left(0, \tau ; V^{*}\right)
$$

if $x_{n} \rightarrow x$ in $W(0, \tau)$ and $u_{n} \rightarrow u$ in $L^{2}(0, \tau ; U)$. Suppose that the level-sets $S_{\alpha}$ are bounded subsets of $X$ and that there exists a locally Lipschitz continuous function $\Phi: X \rightarrow U$, with $\Phi(0)=0$ and such that for all $x \in V$ and $u=-\Phi(x)$

$$
\left\langle G^{\prime}(x), f(x, u)\right\rangle_{V, V^{*}}+f^{0}(x, u) \leq 0
$$

Then $G$ is a global control Lyapunov function.

Proof. (a) Suppose condition (3.2) does not hold. Then there exits an $x_{0} \in V$ such that

$$
\left\langle G^{\prime}\left(x_{0}\right), f\left(x_{0}, u\right)\right\rangle_{V, V *}+f^{0}\left(x_{0}, u\right)>0
$$

for all $u \in U$. Due to assumptions (i) and (ii) the minimal value of the the functional $u \rightarrow\left\langle G^{\prime}\left(x_{0}\right), f\left(x_{0}, u\right)\right\rangle_{V, V *}$ $+f^{0}\left(x_{0}, u\right)$ is attained on $U$ and hence there exists $\epsilon>0$ such that

$$
\left\langle G^{\prime}\left(x_{0}\right), f\left(x_{0}, u\right)+f^{0}\left(x_{0}, u\right) \geq \epsilon>0 \text { for all } u \in U\right.
$$

By convexity of $G$ and (2.5)

$$
\left\langle G^{\prime}(x(t)), \frac{x(t)-x(t-h)}{h}\right\rangle_{V, V *} \leq \frac{G(x(t))-G(x(t-h))}{h} \leq-\frac{1}{h} \int_{t-h}^{t} f^{0}(x, u) \mathrm{d} s .
$$


Since

$$
\begin{aligned}
& \frac{1}{h} \int_{t-h}^{t} f(x(s), u(s)) \mathrm{d} s \rightarrow f(x(t), u(t)) \quad \text { in } V^{*} \\
& \frac{1}{h} \int_{t-h}^{t} f^{0}(x(s), u(s)) \mathrm{d} s \rightarrow f^{0}(x(t), u(t))
\end{aligned}
$$

a.e. $t \in[0, \tau]$ as $t \rightarrow 0^{+}$. Thus, we have

$$
\left\langle G^{\prime}(x(t)), f(x(t), u(t))\right\rangle_{V, V *}+f^{0}(x(t), u(t)) \leq 0
$$

for a.e. $t \in[0, \tau]$. Since $x(t) \rightarrow x_{0}$ in $V$, this contradicts (3.5).

(b) (Sufficiency) Let $\alpha>0$ be arbitrary. By assumption $S_{\alpha}$ is bounded. Hence there exists $\delta>0$ such that $S_{\alpha} \subset B_{\delta}=\left\{x:|x|_{X} \leq \delta\right\}$.

First we prove that the assumptions on $\Phi$ guarantee the existence of a unique locally defined solution $x \in W(0, \tau)$ to

$$
x(t)=x_{0}+\int_{0}^{t} f(x(s),-\Phi(x(s)) \mathrm{d} s,
$$

for every $x_{0} \in B_{\delta}$. Uniqueness is a consequence of the local Lipschitz property of $\Phi$. To verify existence let $x_{0} \in V \cap B_{\delta}$ and set $C=2 \delta$. Define a sequence $x^{k}$ in $W(0, \tau)$ by

$$
x^{k+1}(t)=x\left(t ; x_{0},-\Phi\left(x^{k}\right)\right) .
$$

From the following arguments it follows that $x^{k}$ is well-defined for $\tau$ sufficiently small. In fact let $\tau$ be such that (3.1) admits a unique solution if $\left|x_{0}\right|_{X} \leq C$ and $\int_{0}^{\tau}|u|^{2} \mathrm{~d} s \leq C$. Let $\tau$ be further chosen such that

$$
\sqrt{M_{C}(\tau)\left(\frac{1}{4}+|\Phi|^{2} \tau\right)} \leq 1 \text { and } \max \left(|\Phi|^{2} \tau, \sqrt{\left(M_{C}(\tau) \tau\right)}|\Phi|\right)<1,
$$

where $|\Phi|$ denotes the Lipschitz constant of $\Phi$ on the ball in $X$ with center 0 and radius $C$. If $\left|x^{k}(t)\right|_{X}$ $\leq 2\left|x_{0}\right|_{X}=C$ on $[0, \tau]$, then $u^{k}=-\Phi\left(x_{k}\right)$ satisfies

$$
\int_{0}^{\tau}\left|u^{k}\right|^{2} \mathrm{~d} s \leq C^{2}|\Phi|^{2} \tau
$$

and thus by the local continuous dependence assumption

$$
\left|x^{k+1}(t)\right|_{X} \leq \sqrt{M_{C}(t)\left(\left|x_{0}\right|_{X}^{2}+C^{2}|\Phi|^{2} t\right)} \leq C
$$

on $[0, \tau]$ since $\sqrt{M_{C}(\tau)\left(\frac{1}{4}+|\Phi|^{2} \tau\right)} \leq 1$. If we let $x^{0}(t)=x_{0}$ on $[0, \tau]$ then $x^{k} \in \Sigma=\left\{x \in C(0, \tau ; X):|x(t)|_{X}\right.$ $\leq C$ on $[0, \tau]\}$ for all $k$. For the iterates we find

$$
\left\|x^{k+1}-x^{k}\right\| \leq \sqrt{M_{C}(\tau) \tau}|\Phi||| x^{k}-x^{k-1} \|,
$$

where $\|\cdot\|$ denotes the norm in $C(0, \tau ; X)$. Thus $\left\{x^{k}\right\}$ is a Cauchy sequence in $C(0, \tau ; X)$ since $\sqrt{M(\tau) \tau}|\Phi|<1$. Note that

$$
\left|x^{k+1}-x^{k}\right|_{W(0, \tau)} \leq \sqrt{M_{C}(\tau) \tau}|\Phi||| x^{k+1}-x^{k}||
$$


Thus, there exists $x \in W(0, \tau) \cap \Sigma$ such that $x^{k}$ converges to $x$ strongly in $W(0, \tau)$ and thus $u^{k} \rightarrow u=-\Phi(x)$ as $k \rightarrow \infty$. The continuity condition for $f$ implies that $x \in W(0, \tau)$ is a solution to (3.6).

Let $E$ be a dense subset of Lebesgue points in $(0, T)$ such that for $t \in E$

$$
\lim _{h \rightarrow 0} \frac{1}{h} \int_{t}^{t+h} f(x(s),-\Phi(x(s))) \mathrm{d} s=f(x(t),-\Phi(x(t))) \text { in } V^{*} .
$$

Since $x_{0} \in V$ we have from the general assumptions of this section that $x \in C(0, \tau ; V)$. By convexity of $G$ we obtain

$$
G(x(t+h))-G(x(t)) \leq\left\langle G^{\prime}(x(t+h)), x(t+h)-x(t)\right\rangle_{V, V^{*}} .
$$

By (3.7) and continuity of $x \rightarrow G^{\prime}(x)$ from $V$ to $V$ we have

$$
\frac{\mathrm{d}}{\mathrm{d} t} G(x(t)) \leq\left\langle G^{\prime}(x(t)), f(x(t),-\Phi(x(t)))\right\rangle_{V, V^{*}}, \text { for } t \in E .
$$

Utilizing (3.4) implies that

$$
\frac{\mathrm{d}}{\mathrm{d} t} G(x(t)) \leq-f^{\circ}(x(t),-\Phi(x(t))), \text { for } t \in E .
$$

It follows that

$$
G(x(\tau))+\int_{S}^{\tau} f^{\circ}(x(t),-\Phi(x(t))) \mathrm{d} t \leq G(x(s)),
$$

for $0 \leq s \leq \tau$ and $x_{0} \in V \cap B_{\delta}$. A density argument together with the continuous dependence assumption imply that (3.8) holds for all $x_{0} \in B_{\delta}$. In particular (3.8) holds for $x_{0} \in S_{\alpha}$ and hence a unique global solution to (3.6) exists for every $x_{0} \in S_{\alpha}$ and (2.5) holds for every $T>0$.

\subsection{Quadratic terminal penalty}

In this section we discuss the case when $G(x)=\frac{\alpha}{2}|x|_{X}^{2}, \alpha>0$, can be used as global CLF.

Definition 3.1. The control system (1.1-3.1) is closed-loop dissipative if there exists a locally Lipschitz continuous feedback law $u=-K(x) \in U$ such that

$$
\langle\alpha x, f(x,-K(x))\rangle_{V, V^{*}}+f^{0}(x,-K(x)) \leq 0
$$

for some $\alpha>0$ and all $x \in V$.

If (1.1-3.1) is closed-loop dissipative, then $\frac{\alpha}{2}|x|^{2}$ can serve as a control Lyapunov function for $(2.1,2.2)$ by (3.2) of Theorem 3.1. In general this is not necessarily the case. But we have the following result:

Theorem 3.2. Let $G(x)=\frac{\alpha}{2}|x|^{2}$ and let $V(x)$ and $V_{T}(x)$ be the infinite and the finite horizon value functionals, respectively. We assume that for every $x \in X$ there exists an admissible control $u^{*}(t)=u^{*}(t ; x)$ such that

$$
V\left(x^{*}(T)\right)+\int_{0}^{T} f^{0}\left(x^{*}(t), u^{*}(t)\right) \mathrm{d} t=V(x)
$$

for all $T \geq 0$ and that the corresponding trajectory $x^{*}(t)$ satisfies $\left|x^{*}(t)\right| \leq M \mathrm{e}^{-\omega t}|x|$, for $M \geq 0, \omega>0$ and all $t \geq 0$. Then,

$$
V_{T}(x) \leq V(x)+\frac{M^{2} \alpha}{2} \mathrm{e}^{-2 \omega T}|x|^{2} .
$$


Moreover, if $V(x) \leq \frac{\beta}{2}|x|^{2}$, then

$$
V_{T}(x) \leq\left(\frac{\beta}{2}+\frac{M^{2} \alpha}{2} \mathrm{e}^{-2 \omega T}\right)|x|^{2} \leq\left(\frac{\beta}{\alpha}+M^{2} \mathrm{e}^{-2 \omega T}\right) G(x) .
$$

Proof. Note that

$$
V_{T}(x) \leq \int_{0}^{T} f^{0}\left(x^{*}(t), u^{*}(t)\right) \mathrm{d} t+V\left(x^{*}(T)\right)+G\left(x^{*}(T)\right)-V\left(x^{*}(T)\right) \leq V(x)+G\left(x^{*}(T)\right),
$$

which implies the first assertion. The second assertion simply follows from the first one.

Theorem 3.2 implies that for sufficiently large $\alpha>0$ there exists $\bar{T}>0$ such that for $T \geq \bar{T}$ we have $G(x(T)) \leq V_{T}(x) \leq \rho_{T} G(x)$ with $\rho_{T}<1$ and thus in the notation of Theorem 2.3 we have $G\left(x_{k}\right) \leq \rho_{T}^{k} G\left(x_{0}\right)$, for $k \in \mathbb{N}$.

\subsection{Navier-Stokes equations}

We consider the incompressible Navier-Stokes equations in $\Omega$ : the velocity field $v=v(x, t) \in R^{d}$ and the pressure $p=p(x, t) \in R$ satisfy

$$
v_{t}+v \cdot \nabla v+\operatorname{grad} p=\nu \Delta v+B u(t) \quad \text { and } \quad \operatorname{div} v=0, \quad x \in \Omega, \quad t>0,
$$

with boundary condition $v=0$ on $\Gamma$ and initial condition $v(x, 0)=v_{0}(x)$. Here $\Omega$ is a bounded open domain $R^{d}, d=2,3$ with sufficiently smooth boundary $\Gamma, \nu>0$ is the kinetic viscosity and $B u(\cdot)$ represents the control body force. We use the following standard function spaces (e.g., see [18]). Let $V$ be the divergence-free subspace of $H_{0}^{1}(\Omega)^{d}$ defined by $V=\left\{\phi \in H_{0}^{1}(\Omega)^{d}: \operatorname{div} \phi=0\right\}$ and let $X$ be the completion of $V$ with respect to $L^{2}(\Omega)^{d}$-norm, i.e.

$$
X=\left\{\phi \in L^{2}(\Omega)^{d}: \operatorname{div} \phi=0, \text { and } n \cdot \phi=0 \text { on } \Gamma\right\} .
$$

$X$ is a closed subspace of $L^{2}(\Omega)^{d}$ when equipped by $|\phi|_{X}=|\phi|_{L^{2}}$ as norm. Let $P$ be the orthogonal projection of $L^{2}(\Omega)^{d}$ on $X$. The norm in $V$ is given by $|\phi|_{V}=|\nabla \phi|_{L^{2}}$. We define the Stokes operator $A_{0} \in \mathcal{L}\left(V, V^{*}\right)$ by

$$
\left\langle A_{0} \phi, \psi\right\rangle_{V^{*} \times V}=\sigma(\phi, \psi)=(\nabla \phi, \nabla \psi)_{L^{2}(\Omega)},
$$

for $\phi, \psi \in V$. The operator $A_{0}$ has a closed self-adjoint restriction $\left(A_{0}=-P \Delta\right)$ on $X$ with $\operatorname{dom}\left(A_{0}\right)$ $=H^{2}(\Omega)^{d} \cap V$. Also, define the trilinear form $b$ on $V \times V \times V$ by

$$
b(u, v, w)=\int_{\Omega} u_{i}\left(D_{x_{i}} v_{j}\right) w_{j} \mathrm{~d} x
$$

and assume that $B \in \mathcal{L}(U, X)$. Then (3.9) can be expressed as (3.1) with

$$
\langle f(x, u), \psi\rangle_{V^{*}, V}=\left\langle A_{0} x+B u, \psi\right\rangle_{V^{*}, V}+b(x, x, \psi),
$$

for $x, \psi \in V$ and $u \in U$. The trilinear form $b$ satisfies

$$
b(v, \phi, \psi)+b(v, \psi, \phi)=0
$$


and in the two dimensional case there exist constants $M_{1}, M_{2}$ such that

$$
\begin{aligned}
& |b(v, \phi, \psi)| \leq M_{1}|v|_{H}^{1 / 2}|v|_{V}^{1 / 2}|\phi|_{V}|\psi|_{H}^{1 / 2}|\psi|_{V}^{1 / 2} \\
& |b(v, \phi, \psi)| \leq M_{2}|v|_{H}^{1 / 2}|v|_{V}^{1 / 2}|\phi|_{V}^{1 / 2}|\phi|_{\operatorname{dom}\left(A_{0}\right)}^{1 / 2}|\psi|_{H}
\end{aligned}
$$

for all $v, \phi, \psi \in V$, respectively $\phi \in \operatorname{dom}\left(A_{0}\right)$.

Let us consider the feedback operator $\Phi=\beta B^{*}$, with $\beta>0$. Clearly $\Phi$ satisfies the assumptions of Theorem 3.1(b). With this preparation we have the following:

Proposition 3.1. Assume that $d=2$ and $T>0$ is arbitrary. For every $x_{0} \in X$ and $u \in L^{2}(0, T ; U)$ there exists a unique solution $x \in W(0, T)$ to (3.9), and the continuity property (3.3) holds. If $x_{0} \in V$, then $x \in C(0, T ; V) \cap L^{2}\left(0, T ; \operatorname{dom}\left(A_{0}\right)\right)$. Moreover $G(x)=\frac{\alpha}{2}|x|_{X}^{2}$, with $\alpha>0$ is a control Lyapunov function for the cost-functional $f^{0}(x, u)=\frac{\sigma}{2}\left(|x|^{2}+|u|^{2}\right)$, provided $\sigma$ is sufficiently small.

The first part follows from standard results on Navier-Stokes equations and the second one is a consequence of Theorem 3.1(b) observing that for $u=-\beta B^{*}, \beta>0$

$$
\langle x, f(x, u)\rangle_{V, V^{*}}=-\left\langle A_{0} x, x\right\rangle-\beta\left|B^{*} x\right|^{2},
$$

is negative definite by (3.10).

\section{SEMI-Linear CONTROL SYSTEMS}

Consider the control system of the form

$$
\frac{\mathrm{d}}{\mathrm{d} t} x(t)=A x(t)+F(x(t))+B u(t), \quad x(0)=x_{0} \in X,
$$

where $A$ is the infinitesimal generator of a contraction semigroup $\{S(t): t \geq 0\}$ on $X, B \in \mathcal{L}(U, X)$ and $u \in L_{\text {loc }}^{2}(0, \infty ; U)$. The nonlinear function $F: X \rightarrow X$ is locally Lipschitz in the sense that for each $C \in R$ there exists a constant $k_{C}$ such that

$$
|F(x)|_{X} \leq k_{C} \quad \text { and } \quad|F(x)-F(y)|_{X} \leq k_{C}|x-y|_{X},
$$

for all $x, y \in X$ satisfying $|x|,|y| \leq C$. It can be proved (e.g., see [17]) that for every $x_{0} \in X$ and $u \in$ $L_{\text {loc }}^{2}(0, \infty ; U)$ there exists a unique locally defined mild solution $x$ in $C(0, \tau ; X)$ to $(4.1)$, i.e., there exists $\tau>0$ (depending on $\left|x_{0}\right|$ and $\int_{0}^{\tau}|u|^{2} \mathrm{~d} t$ ) such that

$$
x(t)=S(t) x_{0}+\int_{0}^{t} S(t-s)(F(x(s))+B u(s)) \mathrm{d} s, \text { for } t \in[0, \tau] .
$$

Let $J_{n}=\left(I-\frac{1}{n} A\right)^{-1}$ denote the resolvent of $A$. Then we have the following result:

Theorem 4.1. Assume that $G(x)$ is a $C^{1}$-functional on $X$ satisfying

$$
\left(G^{\prime}(x), A x+F(x)\right)_{X} \leq \omega G(x)+c(x), \quad \text { for all } x \in \operatorname{dom}(A),
$$

for $\omega \in R$ and a continuous function $c: X \rightarrow R$. Then the locally defined solution $x(\cdot)$ to (4.1) satisfies

$$
G(x(t)) \leq \mathrm{e}^{\omega t} G\left(x_{0}\right)+\int_{0}^{t} \mathrm{e}^{\omega(t-s)}\left(c(x(s))+\left(G^{\prime}(x(s)), B u(s)\right)\right) \mathrm{d} s,
$$

for $t \in[0, \tau]$. 
Proof. Let $x_{n}(t)=J_{n} x(t)$. Then $x_{n} \in H^{1}(0, \tau ; X) \cap C(0, \tau ; \operatorname{dom}(A))$ and

$$
\frac{\mathrm{d}}{\mathrm{d} t} x_{n}(t)=A x_{n}(t)+J_{n}(F(x(t))+B u(t)) \text { a.e. in }(0, \tau)
$$

Thus

$$
\frac{\mathrm{d}}{\mathrm{d} t} G\left(x_{n}(t)\right)=\left(G^{\prime}\left(x_{n}(t)\right), A x_{n}(t)+F\left(x_{n}(t)\right)+B u(t)\right)+r_{n}(t)
$$

where

$$
r_{n}(t)=\left(G^{\prime}\left(x_{n}(t)\right), J_{n} F(x(t))-F\left(x_{n}(t)\right)+J_{n} B u(t)-B u(t)\right)
$$

By assumption

$$
\frac{\mathrm{d}}{\mathrm{d} t} G\left(x_{n}(t)\right) \leq \omega G\left(x_{n}(t)\right)+c\left(x_{n}(t)\right)+\left(G^{\prime}\left(x_{n}(t)\right), B u(t)\right)+r_{n}(t),
$$

and using Gronwall's inequality

$$
G\left(x_{n}(t)\right) \leq \mathrm{e}^{\omega t} G\left(x_{0}\right)+\int_{0}^{t} \mathrm{e}^{\omega(t-s)}\left(c\left(x_{n}(s)\right)+\left(G^{\prime}\left(x_{n}(s)\right), B u(s)\right)+r_{n}(s)\right) \mathrm{d} s .
$$

Since $x_{n} \rightarrow x(t)$ in $C(0, \tau ; X)$ and $J_{n} \rightarrow I$ as $n \rightarrow \infty$ we have

$$
\int_{0}^{t} \mathrm{e}^{\omega(t-s)} r_{n}(s) \mathrm{d} s \rightarrow 0 \quad \text { as } n \rightarrow \infty,
$$

and claim follows by taking the limit in (4.3).

Theorem 4.2 (Sufficiency). Suppose that $G$ is a $C^{1}$-functional on $X$ and that $\Phi: X \rightarrow U$ is locally Lipschitz such that

$$
\left(G^{\prime}(x), A x+F(x)-B \Phi(x)\right)_{X} \leq \omega G(x)-f^{0}(x, \Phi(x))+c,
$$

for all $x \in \operatorname{dom}(A)$, where $\omega \in R, c$ is a nonnegative constant and $f^{0}: X \times U \rightarrow R^{+}$is continuous. If $G(x) \geq r\left(|x|_{X}\right)$ for a continuous unbounded function $r: R^{+} \rightarrow R^{+}$, then the closed loop system

$$
\frac{\mathrm{d}}{\mathrm{d} t} x(t)=A x(t)+F(x(t))-B \Phi(x(t)), \quad x(0)=x_{0}
$$

has a unique globally defined mild solution. Moreover, if $\omega \leq 0$ and $c=0$, then $G$ is a global control Lyapunov function for the control system (2.3, 4.1).

Proof. From Theorem 4.1 it follows that

$$
G(x(t)) \leq \mathrm{e}^{\omega t} G(x(0))+\int_{0}^{t} \mathrm{e}^{\omega(t-s)}\left(-f^{0}(x(s), \Phi(x(s)))+c\right) \mathrm{d} s,
$$

for $t \in[0, \tau]$. In particular this implies that

$$
G(x(t)) \leq \mathrm{e}^{\omega t} G(x(0))+c \frac{\mathrm{e}^{\omega t}-1}{\omega}
$$

for $t \in[0, \tau]$. Hence global existence follows from $G(x) \geq r\left(|x|_{X}\right)$ and the continuation method. For $\omega \leq 0$ and $c=0$ the functional $G$ is a CLF by (4.1). 


\subsection{Semi-linear wave equation}

In this subsection we demonstrate the applicability of the above results to the semi-linear wave equation;

$$
y_{t t}+\Psi\left(y_{t}\right)-y_{x x}+y^{3}=u(x, t) \chi_{I}(x), \quad x \in(0,1),
$$

with boundary conditions:

$$
y(t, 0)=0, \quad y_{x}(t, 1)=0,
$$

where $\Psi: R \rightarrow R$ is a Lipschitz continuous function satisfying $\Psi(s) s \geq 0$ for $s \in R$ and $I \subset(0,1)$. To express (4.6) in the abstract form (4.1) we introduce $z(t)=\left(y(t, \cdot), y_{t}(t, \cdot)\right) \in X$ with $X=H_{L}^{1}(0,1) \times L^{2}(0,1)$ where

$$
H_{L}^{1}(0,1)=\left\{\phi \in H^{1}(0,1) \text { with } \phi(0)=0\right\},
$$

equipped with $|\phi|^{2}=\int_{0}^{1}\left|\phi_{x}\right|^{2} \mathrm{~d} x$. Define the linear operator $A$

$$
A(\phi, \psi)=\left(\psi, \phi_{x x}\right)
$$

with

$$
\operatorname{dom}(A)=\left\{(\phi, \psi) \in X: \psi \in H_{L}^{1}(0,1) \text { and } \phi \in H^{2}(0,1), \phi_{x}(1)=0\right\},
$$

and the nonlinear operator

$$
F(\phi)=\left(0,-\phi^{3}-\Psi(\psi)\right) .
$$

Let $B$ be the linear operator on $U=L^{2}(0,1)$ defined by

$$
B u=\left(0, \chi_{I}(x) u\right)
$$

Then (4.6) can be written as (4.1).

Let $u=0$ and define

$$
G(z)=G(\phi, \psi)=\frac{1}{2} \int_{0}^{1}\left(|\psi|^{2}+\left|\phi_{x}\right|^{2}\right) \mathrm{d} x+\frac{1}{4} \int_{0}^{1}|\phi|^{4} \mathrm{~d} x .
$$

Then

$$
\left(G^{\prime}(z), A z+F(z)\right)=-\int_{0}^{1} \Psi(\psi) \psi \mathrm{d} x \leq 0,
$$

for all $z \in \operatorname{dom}(A)$ and hence (4.2) is satisfied with $\omega=c=0$.

Next we consider the linear wave equation

$$
y_{t t}=y_{x x}+u(x, t) \chi_{I}(x), \quad x \in(0,1)
$$

with boundary conditions:

$$
y(t, 0)=0, \quad y_{x}(t, 1)=0,
$$


and aim for establishing (4.4) with

$$
f^{0}(x, u)=\frac{\gamma}{2}\left(|x|_{X}^{2}+|u|_{L^{2}(I)}^{2}\right)
$$

for some $\gamma>0$. Let

$$
G(\phi, \psi)=\int_{0}^{1} \frac{1}{2}\left(|\psi|^{2}+\left|\phi_{x}\right|^{2}\right) \mathrm{d} x+\int_{0}^{1} a(x) \phi_{x} \psi \mathrm{d} x+b \int_{0}^{1} \phi \psi \mathrm{d} x
$$

and $u=-\beta y_{t} \chi_{I}=-\beta \psi \chi_{I}$ where $b$ and $\beta$ are positive constants. For $I=\left(x_{1}, x_{2}\right) \subset(0,1)$ we define the piecewise linear function $a$ on $(0,1)$ by

$$
a(x)=\alpha \begin{cases}x & \text { on }\left[0, x_{1}\right] \\ \frac{x_{2}-x_{1}-1}{x_{2}-x_{1}}\left(x-x_{1}\right)+x_{1} & \text { on }\left(x_{1}, x_{2}\right] \\ x-1 & \text { on }\left(x_{2}, 1\right]\end{cases}
$$

with $\alpha>0$. Then for $z=(\phi, \psi) \in \operatorname{dom}(A)$ we have

$$
\begin{aligned}
J= & \left(G^{\prime}(z), A z-\beta\left(0, \psi \chi_{I}\right)\right)=-\beta \int_{I}|\psi|^{2}+\int_{0}^{1} a(x)\left(\psi \psi_{x}+\phi_{x}\left(\phi_{x x}-\beta \chi_{I} \psi\right)\right) \mathrm{d} x \\
& +b \int_{0}^{1}\left(|\psi|^{2}+\phi\left(\phi_{x x}-\beta \chi_{I} \psi\right)\right) \mathrm{d} x=J_{1}+J_{2}+J_{3} .
\end{aligned}
$$

Here

$$
J_{2} \leq-\int_{0}^{1} \frac{1}{2} a^{\prime}\left(|\psi|^{2}+\left|\phi_{x}\right|^{2}\right) \mathrm{d} x+\beta \alpha|\psi|_{2}\left|\phi_{x}\right|_{2}
$$

and

$$
J_{3} \leq b \int_{0}^{1}\left(|\psi|^{2}-\left|\phi_{x}\right|^{2}\right) \mathrm{d} x+b \beta|\psi|_{2}\left|\phi_{x}\right|_{2}
$$

Note that $a^{\prime}=\alpha\left(1-\frac{1}{x_{2}-x_{1}}\right)<0$ on $I$ and on $a^{\prime}=\alpha>0$ on $I^{c}$. Assume that $x_{2}-x_{1}>\frac{1}{2}$ and let $b=\frac{\alpha}{4\left(x_{2}-x_{1}\right)}$. For $c=\left(\frac{1}{2}-\frac{1}{4\left(x_{2}-x_{1}\right)}\right) \alpha>0$, we have $b+\frac{a^{\prime}}{2}=c$ on $I$ and $\frac{1}{2} a^{\prime}-b=c$ on $I^{c}$. Thus, if $\beta=b+c-\frac{a^{\prime} \mid I}{2}=\frac{\alpha}{2\left(x_{2}-x_{1}\right)}=2 b$, then

$$
J \leq-c \int_{0}^{1}\left(|\psi|^{2}+\left|\phi_{x}\right|^{2}\right)+\beta \alpha|\psi|_{2}\left|\phi_{x}\right|_{2}+b \beta|\psi|_{2}\left|\phi_{x}\right|_{2} .
$$

Hence we can select $0<\alpha<1$ such that

$$
J \leq-\frac{c}{2} \int_{0}^{1}\left(|\psi|^{2}+\left|\phi_{x}\right|^{2}\right) \mathrm{d} x .
$$

Further $\alpha>0$ can be chosen such that

$$
k_{1}\left(\left|\phi_{x}\right|^{2}+|\psi|^{2}\right) \leq G(\phi, \psi) \leq k_{2}\left(\left|\phi_{x}\right|^{2}+|\psi|^{2}\right),
$$


for positive constants $k_{1}, k_{2}$ independent of $(\phi, \psi) \in X$. From these estimates Theorem 4.2 is applicable and we have:

Proposition 4.1. If $\alpha, \beta, b, \gamma$ are sufficiently small positive constants and $\frac{1}{2}<x_{2}-x_{1}$, then $G$ defined in (4.9) is a control Lyapunov function for the linear system (4.7) with cost given by (4.8) and feedback law $u=-\beta y_{t} \chi_{I}$. Moreover (4.4) holds with $\omega<0$ and $c=0$.

We now turn to the nonlinear equation (4.6).

Proposition 4.2. Assume $\frac{1}{2}<x_{2}-x_{1}$ and $|\Psi(\psi)| \leq d|\psi|$ with $d \geq 0$ sufficiently small. Then there exists positive constants $\alpha, b$ such that $G$ defined by

$$
G(z)=\frac{1}{2} \int_{0}^{1}\left(|\psi|^{2}+\left|\phi_{x}\right|^{2}\right) \mathrm{d} x+\frac{1}{4} \int_{0}^{1}|\phi|^{4} \mathrm{~d} x+\int_{0}^{1} a(x) \phi_{x} \psi \mathrm{d} x+b \int_{0}^{1} \phi \psi \mathrm{d} x
$$

is a control Lyapunov function for the nonlinear system (4.6) with cost given by (4.8) and feedback law $u=$ $-\beta y_{t} \chi_{I}$.

Proof. We show that (4.4) holds with $\omega=0$ and $c=0$. If $J$ is defined as in (4.10), then

$$
\begin{aligned}
& \left(G^{\prime}(z), A x+F(z)-\beta\left(0, \psi \chi_{I}\right)\right)_{X}= \\
& \quad \leq J+\int_{0}^{1} a(x) \phi_{x}\left(-\phi^{3}-\Psi(\psi)\right) \mathrm{d} x+b \int_{0}^{1} \phi\left(-\phi^{3}-\Psi(\psi)\right) \mathrm{d} x .
\end{aligned}
$$

where $J$ is defined as in (4.10). Here

$$
-\int_{0}^{1}\left(a(x) \phi_{x} \phi^{3}+b \phi^{4}\right) \mathrm{d} x=\int_{0}^{1}\left(\frac{1}{4} a^{\prime}-b\right) \phi^{4} \mathrm{~d} x \leq 0,
$$

for $b \geq \frac{\alpha}{4}$ since $a^{\prime} \leq \alpha$. Thus, the proposition follows from (4.11, 4.12).

\section{Local CONTROL LyapunOV FUnCtion}

In this section we describe a method for construction of local control Lyapunov functions based on the Lyapunov equation. Consider the semi-linear control system

$$
\frac{\mathrm{d}}{\mathrm{d} t} x(t)=A x(t)+F(x(t))+B u(t), \quad x(0)=x_{0} \in X,
$$

with $F$ locally Lipschitz continuous. Assume that $A-\beta B B^{*}, \beta>0$, generates an exponentially stable $C_{0^{-}}$ semigroup $S(t)$ on $X$. Let $Q$ denote a nonnegative, self-adjoint operator on $X$, and let $\Sigma$ be the bounded, nonnegative self-adjoint solution to the Lyapunov

$$
2\left(\left(A-\beta B B^{*}\right) x, \Sigma x\right)_{X}+(Q x, x)=0 \quad \text { for all } x \in \operatorname{dom}(A),
$$

that is, $\Sigma x=\int_{0}^{\infty} S^{*}(t) Q S(t) x \mathrm{~d} t$, for $x \in X$.

We define

$$
G(x)=\frac{1}{2}\left(|x|^{2}+(\Sigma x, x)\right), \text { for } x \in X,
$$


and set $u=-\beta B^{*} x$. For this choice of $u$ there exists a unique local solution $x(\cdot)$ of $(5.1)$ on $[0, \tau], \tau>0$. Assume that there exists a continuous function $\gamma: X \rightarrow R$ such that

$$
(A x, x)-\beta\left|B^{*} x\right|^{2}+(x, F(x))-\frac{1}{2}(Q x, x)+(F(x), \Sigma x) \leq \gamma(x),
$$

for all $x \in \operatorname{dom}(A)$. Then

$$
G(x(t)) \leq G\left(x_{0}\right)+\int_{0}^{t} \gamma(x(s)) \mathrm{d} s \text { for } s \in[0, \tau] .
$$

Assume further that there exists $\alpha>0$ such that

$$
\gamma(x)+f^{0}\left(x,-\beta B^{*} x\right) \leq 0, \text { for all } x \in S_{\alpha} .
$$

Then (5.1) with $u=-\beta B^{*} x$ admits a global solution for $x_{0} \in S_{\alpha}$ and $G$ is a local control Lyapunov function for (1.1) subject to (5.1).

\subsection{Example}

As an example consider the controlled reaction-diffusion system

$$
y_{t}(t, x)=D y_{x x}(t, x)+f(y)+\sum_{i=1}^{m} b_{i}(x) u_{i}(t), \text { for } t>0, x \in(0,1)
$$

with boundary conditions $y(t, 0)=y(t, 1)=0$, where $y: R^{+} \times[0,1] \rightarrow R^{d}, D$ is a positive diagonal matrix, $f: R^{d} \rightarrow R^{d}$ is the reaction rate and $b_{i} \in L^{\infty}\left((0,1) ; R^{d}\right)$ is the $i$-th control distribution function.

Let $X=L^{2}\left((0,1) ; R^{d}\right), U=R^{m}$ and set

$$
\begin{gathered}
A y=D y_{x x} \quad \text { with } \operatorname{dom}(A)=\left\{y \in X: y_{x}, y_{x x} \in X: y(0)=y(1)=0\right\}, \\
{[F(y)](x)=f(y(x)),} \\
{[B u](x)=\sum_{i=1}^{m} b_{i}(x) u_{i}, \text { for } x \in(0,1) .}
\end{gathered}
$$

If $F$ is locally Lipschitz in the sense of Section 4 , with $F(0)=0$, and, for example, $Q=\delta I$, with $\delta>0$, and $f^{0}$ is quadratic, then it is straightforward to check condition (4.4) with $c=0$.

Alternatively one can utilize the Gelfand triple formulation of (5.3) with $V=H_{0}^{1}\left((0,1) ; R^{d}\right)$. The left hand side of (5.3) becomes

$$
-\left(D y_{x}, y_{x}\right)-\beta\left|B^{*} y\right|^{2}-\frac{1}{2}(Q y, y)+(F(y), y+\Sigma y)
$$

which suggests to introduce an equivalent norm $\|y\|$ on $V$ defined by

$$
\|y\|^{2}=\left(D y_{x}, y_{x}\right)+\beta\left|B^{*} y\right|^{2}+\frac{1}{2}(Q y, y)
$$

Suppose that $F$ satisfies

$$
\left|(F(y), y+\Sigma y)_{X}\right| \leq\|y\|^{2} q(|y|), \text { for all } y \in X
$$


for some $q: R^{+} \rightarrow R$. Then, if there exists $\alpha>0$ such that

$$
(q(|y|)-1)\|y\|^{2}+f^{0}\left(y,-B^{*} y\right) \leq 0 \text { for all } y \in S_{\alpha},
$$

equation (5.4) is satisfied with $\gamma(y)=(q(|y|)-1)\|y\|^{2},(5.5)$ admits a global solution for all $y(0) \in S_{\alpha}$, and $G$ is a local control Lyapunov function.

\subsection{Approximation}

Let $X_{n}$ be finite dimensional subspaces of $X$ with $\bigcup_{n=1}^{\infty} X_{n}=X$, and denote by $P_{n}$ the orthogonal projections from $X$ onto $X_{n}$. Let $\left\{A_{n}\right\}$ be a sequence of approximations of $A$ on $X_{n}$ satisfying

$$
\left(A_{n} P_{n} x, x\right)_{X} \leq 0, \quad \text { for all } x \in X,
$$

and

$$
\left|\left(I-A_{n}\right)^{-1} P_{n} x-(I-A)^{-1} x\right|_{X} \rightarrow 0, \quad \text { as } n \rightarrow \infty
$$

for all $x \in X$. By the Trotter-Kato theorem

$$
\left|\mathrm{e}^{A_{n} t} P_{n} x-S(t) x\right|_{X} \rightarrow 0, \quad \text { as } n \rightarrow \infty
$$

for all $x \in X$, uniformly on bounded $t$-intervals. Let $B_{n}=P_{n} B$ and $Q_{n}=P_{n} Q P_{n}$. We further assume that

$$
\left|\mathrm{e}^{\left(A_{n}-\beta B_{n} B_{n}^{*}\right) t} P_{n} x\right| \leq M \mathrm{e}^{-\omega t}|x|
$$

for constants $M \geq 1$ and $\omega>0$ independent of $x \in X$. Let $\Sigma_{n}: X_{n} \rightarrow X_{n}$ satisfy the Lyapunov equation on $X_{n}$

$$
\left(A_{n}-\beta B_{n} B_{n}^{*}\right)^{*} \Sigma_{n}+\Sigma_{n}\left(A_{n}-\beta B_{n} B_{n}^{*}\right)+Q_{n}=0 .
$$

That is,

$$
\Sigma_{n}=\int_{0}^{\infty} \mathrm{e}^{\left(A_{n}-\beta B_{n} B_{n}^{*}\right)^{*} t} Q_{n} \mathrm{e}^{\left(A_{n}-\beta B_{n} B_{n}^{*}\right) t} \mathrm{~d} t .
$$

From our assumptions it follows that, $\Sigma_{n} P_{n} x \rightarrow \Sigma x$ as $n \rightarrow \infty$ for all $x \in X$. We construct a sequence of functions $G_{n}(x)$ on $X$ by

$$
G_{n}(x)=\frac{1}{2}\left(|x|^{2}+\left(\Sigma_{n} P_{n} x, P_{n} x\right)\right)
$$

Note that

$$
\left(A x-\beta B B^{*} x, \Sigma_{n} P_{n} x\right)=-\frac{1}{2}\left(Q_{n} x, x\right)+\left(A x-A_{n} P_{n} x, \Sigma_{n} P_{n} x\right)-\beta\left(B B^{*} x-B_{n} B_{n}^{*} x, \Sigma_{n} P_{n} x\right) .
$$

Suppose condition (5.3) holds. Then we have

$$
G_{n}(x(t)) \leq G_{n}\left(x_{0}\right)+\int_{0}^{t}\left(\gamma(x(t))+e_{n}(x(t))\right) \mathrm{d} t,
$$


where

$$
e_{n}(x)=\frac{1}{2}\left(\left(Q-Q_{n}\right) x, x\right)+\left(A x(t)-A_{n} P_{n} x, \Sigma_{n} P_{n} x\right)-\beta\left(B B^{*} x-B_{n} B_{n}^{*} x, \Sigma_{n} P_{n} x\right)-\left(F(x), \Sigma x-\Sigma_{n} P_{n} x\right)
$$

Here, we note that

$$
\left(A x-A_{n} P_{n} x, \Sigma_{n} P_{n} x\right)=0,
$$

if $X_{n}$ and $X_{n}^{\perp}$ are invariant subspaces of $A$. Hence, if for some $\alpha_{n}>0$

$$
\gamma(x)+e_{n}(x)+f^{0}\left(x,-\beta B^{*} x\right) \leq 0
$$

for all $x \in S_{\alpha_{n}}^{n}=\left\{x: G_{n}(x) \leq \alpha_{n}\right\}$ then $G_{n}$ is a local control Lyapunov function for the infinite dimensional control problem (1.1), (5.1).

Remark. Alternatively to the semi-linear formulation used above, the approximation procedure can be cast in the Gelfand triple formulation.

Example. Consider the controlled reaction-diffusion system as in Section 5.1. Assume that $D$ is nonsingular, $Q$ is compact and (5.4) holds. Let $X_{n}=\operatorname{span}\left\{\phi_{i}\right\}_{i=1}^{n}$ where $\phi_{i}$ is the $i$-th eigen function of $A$. Since $\left\|\Sigma_{n}-\Sigma\right\| \rightarrow 0$ and

$$
e_{n}(x)=\frac{1}{2}\left(\left(Q-Q_{n}\right) x, x\right)-\beta\left(B B^{*}-B_{n} B_{n}^{*} x, x\right)+\left(F(x), \Sigma x-\Sigma_{n} x\right),
$$

there exists an $\alpha_{n}>0$ such that (5.7) is satisfied. Moreover, $\left\{\phi_{i}\right\}$ being eigenfunctions can be replaced by any complete basis in $\left(H_{0}^{1}\right)^{d}$ since in general we can show

$$
\left(A x-A_{n} P_{n} x, \Sigma_{n} P_{n} x\right) \leq \epsilon_{n} \sqrt{(A x, x)}|x|
$$

with $\epsilon_{n} \rightarrow 0$ as $n \rightarrow \infty$ for $x \in \operatorname{dom}(A)$.

\section{REFERENCES}

[1] F. Allgöwer, T. Badgwell, J. Qin, J. Rawlings and S. Wright, Nonlinear predictive control and moving horizon estimation an introductory overview, Advances in Control, edited by P. Frank. Springer (1999) 391-449.

[2] T.R. Bewley, Flow control: New challenges for a new Renaissance. Progr. Aerospace Sci. 37 (2001) 21-58.

[3] H. Chen and F. Allgöwer, A quasi-infinite horizon nonlinear model predictive control scheme with guaranteed stability. Automatica 34 (1998) 1205-1217.

[4] H. Choi, M. Hinze and K. Kunisch, Instantaneous control of backward facing step flow. Appl. Numer. Math. 31 (1999) 133-158.

[5] H. Choi, R. Temam, P. Moin and J. Kim, Feedback control for unsteady flow and its application to the stochastic Burgers equation. J. Fluid Mech. 253 (1993) 509-543.

[6] R.A. Freeman and P.V. Kokotovic, Robust Nonlinear Control Design, State-Space an Lyapunov Techiques. Birkhäuser, Boston (1996).

[7] W.H. Fleming and M. Soner, Controlled Markov Processes and Viscosity Solutions. Springer-Verlag, New York (1993).

[8] C.E. Garcia, D.M. Prett and M. Morari, Model predictive control: Theory and practice - a survey. Automatica 25 (1989) $335-348$.

[9] M. Hinze and S. Volkwein, Analysis of instantaneous control for the Burgers equation. Nonlinear Analysis TMA (to appear).

[10] K. Ito and K. Kunisch, On asymptotic properties of receding horizon optimal control. SIAM J. Control Optim (to appear).

[11] A. Jadababaie, J. Yu and J. Hauser, Unconstrained receding horizon control of nonlinear systems. Preprint.

[12] D.L. Kleinman, An easy way to stabilize a linear constant system. IEEE Trans. Automat. Control 15 (1970) $692-712$.

[13] D.Q. Mayne and H. Michalska, Receding horizon control of nonlinear systems. IEEE Trans. Automat. Control 35 (1990) $814-824$. 
[14] V. Nevistič and J. A. Primbs, Finite receding horizon control: A general framework for stability and performance analysis. Preprint.

[15] J.A. Primbs, V. Nevistič and J.C. Doyle, A receding horizon generalization of pointwise min-norm controllers. Preprint.

[16] P. Scokaert, D.Q. Mayne and J.B. Rawlings, Suboptimal predictive control (Feasibility implies stability). IEEE Trans. Automat. Control 44 (1999) 648-654.

[17] F. Tanabe, Equations of Evolution. Pitman, London (1979).

[18] R. Temam, Navier-Stokes Equations, Theory and Numerical Analysis. North Holland, Amsterdam (1984). 